\title{
POTENCIAL TURISTICO DE CATARINA
}

Alfonso Jirón García

\section{Resumen}

Catarina es una ciudad que forma parte de los pueblos blancos, localizados en el contorno de la Laguna de Apoyo.

Este estudio que se presenta tiene como objetivo la identificación del potencial turístico de Catarina a partir de la variedad de recursos naturales y culturales que presenta el territorio. Para su realización se hicieron reiteradas visitas a la localidad, consultas bibliográficas, entrevistas a líderes comarcales y a responsables de las instituciones, además se aplicaron encuestas a los turistas.

Catarina está localizada a $6 \mathrm{~km}$ de Masaya en el sector noroccidental de la Laguna de Apoyo. Tiene como principal atractivo la Reserva Natural Laguna de Apoyo. Esta Reserva la conforman la misma Laguna, la variedad de flora y de fauna, las altas pendientes, y la espesa vegetación para practicar el ecoturismo.

El cráter de La Laguna Apoyo se formó hace unos 23000 años, su belleza paisajística la convierten en un destino muy interesante para los turistas nacionales y extranjeros que desean estar en contacto con la naturaleza.

Para los recursos culturales, el potencial turístico de Catarina está representada por la iglesia católica, con una arquitectura colonial, además cuenta con centros artesanales que trabajan la madera, el barro y las fibras vegetales. De igual manera cuenta con numerosos jardines, viveros y un impresionante mirador.

Completan el potencial turístico de la ciudad la infraestructura, sus restaurantes y hoteles, localizados en la ciudad, al borde del mirador y a la orillas de la Laguna. Las actividades turísticas implementadas son: el senderismo, el turismo de aventura la natación y el ecoturismo.

Palabras claves: Recursos turísticos, Trópico seco, Reserva Natural, Ecoturismo, Patrimonio turístico.

\begin{abstract}
Catarina is a small town located within "Los pueblos blancos", placed around the Apoyo Lagoon.

This research aims to identify the tourist potential from the variety of natural and cultural resources posing the territory. There were performed many visits to the town, literature survey, interviews with community leaders and institutional responsible, and tourists surveys. Catarina is located $6 \mathrm{~km}$. from Masaya, to the northwest sector of the Apoyo Lagoon. Its main attraction is the Lagoon's natural reserve. This reserve consists of the lagoon itself, variety of flora, fauna, high slopes and canopy ideal for ecotourism. The crater lagoon was formed about 23,000 years ago, and its beauty landscape make it a tourist destination for national and foreign that want to get in touch with nature.

Cultural resources is represented by the catholic church's colonial architecture; besides other artisanal places that manufacturing wood, mud, and vegetable fibres. Similarly, there are many gardens, nurseries and an impressive viewpoint.

Completing tourist potential of the town, infrastructure, restaurants and hotels surrounding the viewpoint and river of lagoon. Tourist activities implemented are hiking, swimming, ecotourism, and adventure.
\end{abstract}

Keywords: tourist resources, Natural reserve, Ecotourism, tourism heritage. 


\section{Introduccion}

$\mathrm{E}$ potencial de recursos naturales que tienen las diferentes regiones del país, es variado debido a que por los cuatro puntos cardinales se encuentran manifestaciones paisajísticas tanto en el orden cultural como natural.

Los componentes turísticos que Nicaragua ofrece al mundo son: Los ríos, lagunas, lagos, playas vírgenes, islas, volcanes y las reservas naturales así como los monumentos, iglesias, museos etc. Constituyen todo un conjunto de atractivos turísticos que son objetos de admiración por el turista, es por esta razón que decimos que Nicaragua es un país por descubrir.

Para el estudio se ha tomado como punto de referencia el departamento de Masaya que además de ser la cuna del folklore nicaragüense presenta diversidades paisajísticas, destacándose entre ellos el municipio de Catarina quien constituyendo una localidad muy pequeña, cuenta con una variedad de factores ambientales, urbanísticos y climatológicos y que por su posición geográfica lo hacen destacarse entre las otras localidades aledañas a la laguna, convirtiéndose en un destino turístico de referencia.

En el presente artículo se presenta un breve detalle de los resultados obtenidos como producto de las reiteradas visitas de campo, de la aplicación de encuestas, de la realización de entrevistas. Además de las observaciones realizadas sobre la actividad turística del lugar.

El trabajo tiene el atractivo de presentan los sitios turísticos de la localidad destacando la impresionante laguna de Apoyo, la belleza de sus viveros y jardines, los talleres artesanales y sus obras de artes. De igual manera se presenta la infraestructura hotelera que se desarrolla.

Otro interés del estudio, está relacionado con la presentación de algunas estrategias que permitan la creación de más infraestructuras y servicios turísticos, diseño de senderos turísticos alrededor de la laguna. Todo esto con el propósito de brindar mejores condiciones a los visitantes y para hacer una explotación racional y sostenible de los recursos que posee.

\section{Material y Métodos}

Para la realización del estudio se procedió de la siguiente manera:

- Se hicieron visitas a la localidad de Catarina y a las diversas comarcas aledañas con el propósito de conocer los componentes paisajísticos que presenta cada una de ellas, esto sirvió también para interrelacionarnos con los pobladores, principalmente con los líderes comunales. Durante esta fase se trabajo con el GPS para obtener los primeros datos que posteriormente servirían para el mapeo.

Se procedió a las revisiones de documentos para detectar los trabajos realizados y que están relacionados con las actividades turísticas y de los cuales pudiéramos obtener alguna información preliminar para nuestro estudio.

- Se hicieron entrevistas exploratorias a los líderes comarcales, a los guarda parques y a los responsables de instituciones.

- Se hizo el reconocimiento del estado de infraestructura de: caminos, carreteras, hoteles, restaurantes así como el estado de la flora y la fauna. 
- Se aplicaron encuestas a diferentes comunidades que están en los alrededor de la laguna tanto en el área rural como urbana.

- Se realizaron entrevistas a turistas nacionales e internacionales con la intención de obtener una valoración relacionada al potencial turístico de Catarina.

El estudio presenta las características descriptivas analíticas. Los métodos deductivos utilizados se emplearon para la recolección de información bibliográfica y el método inductivo aplicado de forma directa cuando se hicieron las giras de campos hacia la localidad en estudio.

Un componente importante para la recolección de información lo constituyeron los estudiantes de $\mathrm{V}$ año de la carrera de geografía quienes representan el apoyo fundamental de esta investigación.
Con la información obtenida, la siguiente tarea consistió en la transcripción de las entrevistas realizadas. En la elaboración de la matriz de datos producto de todas las encuestas fueron procesadas y analizadas con el programa estadístico SPSS.

También es importante señalar que se emplearon algunos instrumentos para la obtención de los datos como: guías de encuestas socio económico y ambiental, guías de entrevistas a turistas, guía de entrevistas a líderes comarcales, guarda parques y guías de observación.

\section{Localizacion Geográfica}

El municipio de Catarina forma parte del departamento de Masaya, se encuentra localizado $6 \mathrm{~km}$ de la cabecera de este departamento y a $35 \mathrm{Km}$. de la ciudad de Managua; está ubicado en el sector noroccidental de la laguna de Apoyo en la región de los Pueblos Blancos. Sus coordenadas son $11^{\circ} 54^{\prime}$ Latitud Norte y $86^{\circ} 04^{\prime}$ Longitud Oeste.

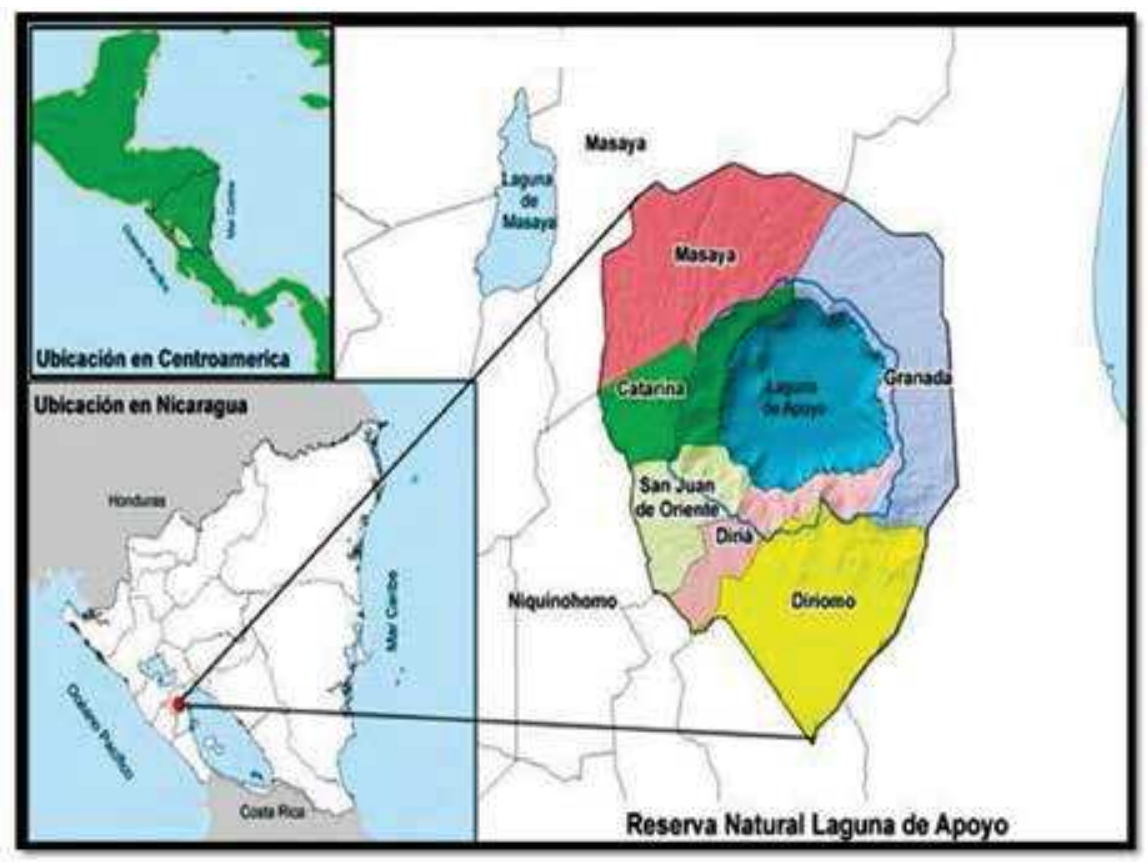

Mapa 1. Reserva Natural Laguna de Apoyo. 


\section{Potencial Turístico de Catarina}

\section{Los recursos turísticos}

Los recursos turísticos pueden ser de carácter natural y cultural, implicando un atractivo para los visitantes y a la vez hacen posible la actividad turística porque satisfacen las necesidades de los demandantes.

\section{Recursos Naturales}

Son todos aquellos espacios naturales con características muy representativas del municipio, que son en sí motivo suficiente para emprender un viaje y que son parte del patrimonio turístico municipal, entre ellos se tienen: las reservas naturales, los parques naturales, el paisaje, la flora, la fauna, las condiciones climáticas, los mares, los ríos, los lagos, las lagunas, los esteros, las cascadas, los ojos de agua, los manantiales, las montañas, y los miradores entre otros. (INTUR: 2007; 10)

En el municipio se encuentran como principales atractivos naturales: $\mathrm{La}$ Reserva Natural de Apoyo y dentro de ella se encuentra la Laguna de Apoyo.

\section{Reserva Natural de Apoyo}

La Reserva y la laguna de Apoyo contienen gran variedad de flora y fauna, además de fuentes hídricas y lugares históricos

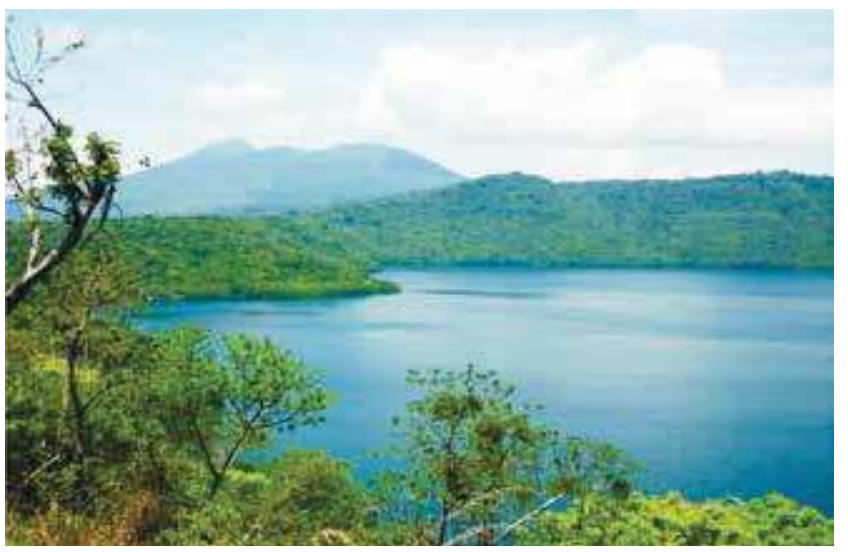

Foto 1. Reserva Natural Laguna de Apoyo. que convierten a esta reserva natural en un sitio con excelentes condiciones para desarrollar el ecoturismo.

A partir del 1991 el sitio fue declarado como Reserva Natural Laguna de Apoyo. Tanto la laguna como el interior del cráter se encuentran divididos entre los departamentos de Granada y Masaya. Los sectores norte y oeste están dentro de los municipios masayenses de Catarina y San Juan de Oriente.

Los municipios granadinos de Diriá y Granada, ocupan los sectores sur y este de la reserva. Otros municipios con influencia por encontrarse en la zona de amortiguamiento son Diriomo (Departamento de Granada) y en menor grado Niquinohomo (Departamento de Masaya).

Para el ingreso a la zona, hay dos entradas principales que nos conducen al núcleo de la reserva (es decir hasta la laguna). Una está ubicada en el municipio de Catarina (Masaya) y la segunda entrada está en el municipio de Diriá (Granada). También hay diversos senderos desde todos los sectores del borde del cráter que bajan hasta la laguna.

Aunque no se cuentan con cifras precisas, según la Alcaldía municipal de Catarina se

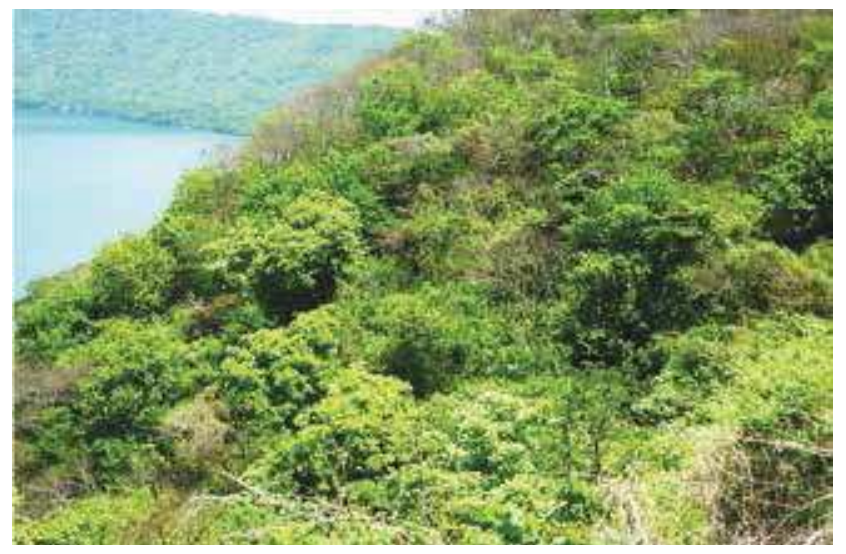

Foto 2. Bosque de la Reserva. 
estima que anualmente la Reserva Natural Laguna de Apoyo recibe entre 30,000 y 60,000 turistas nacionales y extranjeros.

Los bosques de Apoyo contienen numerosas especies de plantas y árboles de trópico seco, así como variedad y cantidad de bellas orquídeas.

\section{Laguna de Apoyo}

La Laguna de Apoyo tiene una extensión territorial de 3,500 hectáreas que pertenecen al Sistema Nacional de Áreas Protegidas de Nicaragua. Está rodeada por dos ciudades principales Masaya y Granada y tres municipios: Diriá, San Juan de Oriente y Catarina.

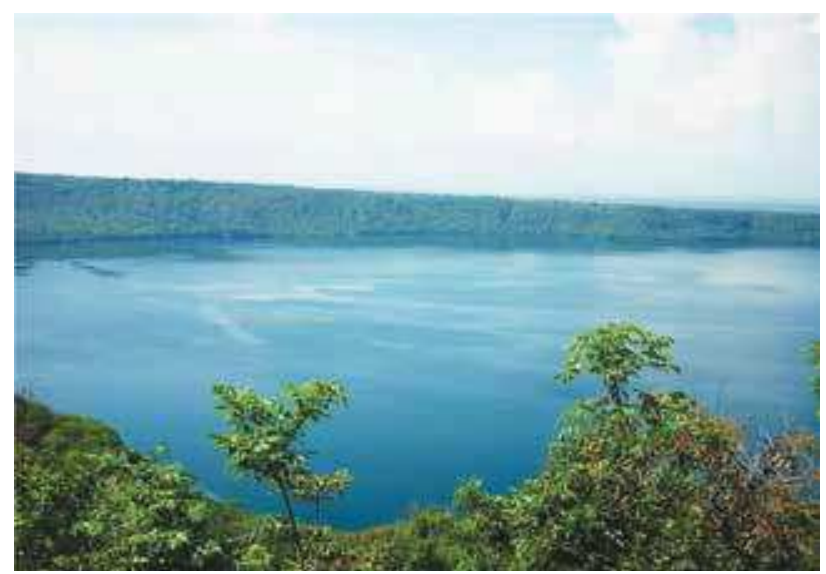

Foto 3. Paisaje de Laguna Apoyo.

El cráter de Apoyo se originó hace aproximadamente 23,000 años, luego de una potentísima explosión volcánica que dejó un hoyo de seis kilómetros de diámetro.

Con el pasar del tiempo, la afluencia de aguas subterráneas y el aporte de las lluvias permitieron el surgimiento de la laguna, y las laderas se cubrieron de abundante vegetación. En la actualidad, este cráter es considerado un volcán durmiente, en reposo, con cierta actividad lo que se manifiesta a través de algunas fumarolas en su interior.
Sus aguas son muy profundas: según estudios, el fondo del hoyo cónico está a más de $200 \mathrm{~m}$. de profundidad y la orilla de la laguna se encuentra a 75 m.s.n.m. Aunque no son aptas para el consumo humano, son también aguas muy limpias, con bajos niveles de contaminación.

La altura máxima de las laderas alcanza los 623 m.s.n.m. y está ubicada en el municipio de Catarina, departamento de Masaya.

La belleza paisajística del sitio, la naturaleza de abundante flora y fauna, las tranquilas y limpias aguas de la laguna y el amplio anillo boscoso de las laderas hacen de Apoyo un destino increíble para visitantes nacionales y extranjeros, con una interesante oferta de actividades turísticas.

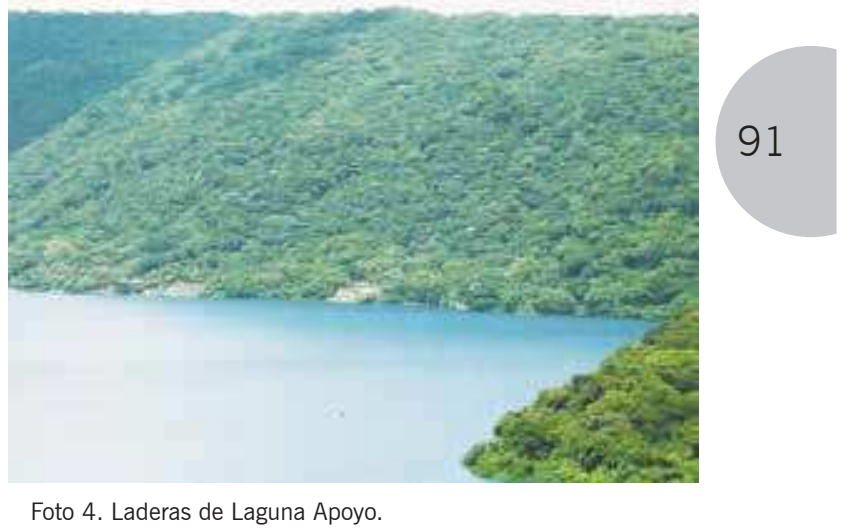

\section{Recursos Culturales}

Se consideran recursos culturales a las diferentes expresiones culturales tangibles e intangibles que presenta en municipios como: lugares arqueológicos, paleontológicos, sitios históricos y religiosos, productos artesanales, monumentos, curiosidades, lugares para visitas guiadas, así como atracciones y manifestaciones tradicionales, costumbres, gastronomía y las manifestaciones folklóricas, entre otras. (INTUR: 2007; 10). 


\section{Iglesia Santa Catalina}

La iglesia con rasgos coloniales fue construida en el año 1800 y fue consagrada a "La Virgen Mártir de Santa Catalina. Es un templo asentada sobre una pequeña loma que domina toda la ciudad, es famosa por las actividades religiosas en honor a su santa patrona (Entrevista a funcionarios de la alcaldía).

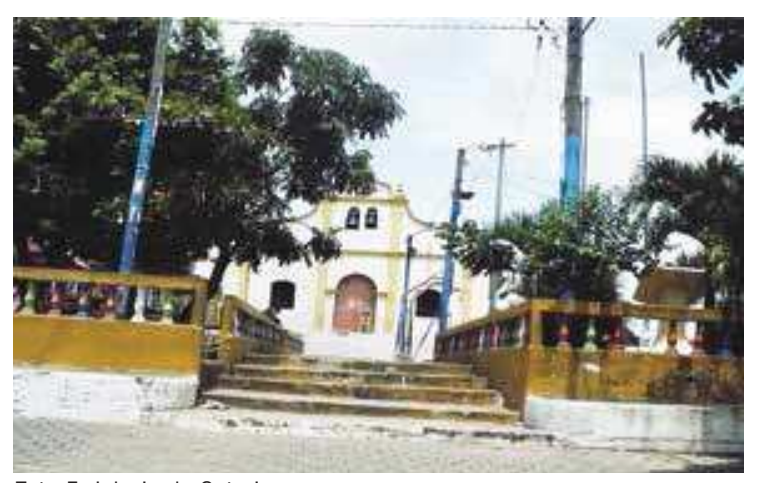

Foto 5. Iglesia de Catarina.

\section{Artesanía en Madera}

En la ciudad se encuentran numerosos talleres de muebles en donde se realizan trabajos finos, elegantes y rústicos de madera sólida donde se destacan camas, roperos, sillas, armarios, baúles, mesas de centro, y los famosos caballitos labrados a mano.

\section{Artesanía en Barro}

En Catarina se elaboran ollas, maceteras, y comales utilizando barro o arcilla, generalmente la artesanía es fabricada en San Juan de Oriente, pero es pintada y comercializada en Catarina.

\section{Artesanía en Bambú}

En Catarina se contabilizan los talleres familiares artesanales que elaboran muebles y ornamentos de gran variedad, como canastos adornos y maceteras.

\section{Jardines}

Catarina cuenta con 60 jardines. Los jardines se encuentran a lo largo de la carretera próxima a la entrada del poblado y también están ubicados en las calles principales de la ciudad hasta llegar al mirador. Son lugares que se caracterizan por el aroma, la diversidad y la belleza de las flores y plantas, los cuales son aprovechados por los visitantes quienes atraídos por el colorido de las flores acceden a comprar cualquier tipo de planta para embellecer sus hogares.

\section{Viveros}

Vivero es un conjunto de instalaciones que tiene como propósito fundamental la producción de plantas. Es una actividad agroeconómica, confinada a espacios reducidos, que incluye plantas ornamentales, plantas medicinales, árboles frutales injertados y árboles forestales en estado de crecimiento y desarrollo.

Esta actividad en el municipio de Catarina, es una de las principales actividades económicas locales y representa un fuerte atractivo turístico porque no solo genera beneficios económicos a la comunidad, sino también ambientales.

Muchas de las plantas cultivadas en los viveros como musgos, helechos, líquenes y crisantemos sirven para exportarlas a países europeos, a los E.E.U.U. y a Centroamérica.

\section{Recursos Creados por el Hombre Mirador de Catarina}

Entre los recursos creados por el hombre se encuentra el Mirador de Catarina.

Catarina es famosa por su majestuosa vista a la laguna de Apoyo, así como por sus viveros.

El mirador de Catarina está en una de las colinas más altas (500 metros) rodeando la Laguna de Apoyo, y con una hermosa 
vista paisajística. Desde este sitio se puede observar toda la laguna, así como el Lago de Nicaragua que se encuentra detrás de ella, de igual forma se observa el Volcán Mombacho que está en parte rodeado por las aguas del majestuoso lago Cocibolca.

El mirador es muy popular y es visitado por los turistas nicaragüenses y extranjeros durante los fines de semana cuando los amigos y familiares se reúnen para apreciar la vista y disfrutar del agradable clima. Hay también algunas tiendas de suvenires en el mirador, en donde puede comprar variedad de artesanías. Todos estos componentes presentes en el mirador forman parte de la oferta turística estratégica que ofrece Catarina.

\section{Mirador Diriomito}

Este sitio está ubicado en la comunidad de Diriomito, sobre la carretera que va desde Pacaya hacia la Laguna, el lugar es muy accesible ya que la carretera está completamente adoquinada y en buen estado; desde este lugar puede observarse de una forma muy impresionante la Laguna de Apoyo.

El mirador está equipado con algunos restaurantes que ofrecen deliciosos platillos a sus visitantes, muy cerca también se encuentra ubicado un establecimiento para la práctica de parapente.

\section{Servicios Turísticos}

Los servicios turísticos, son el conjunto de bienes y servicios con que cuenta el municipio, los cuales facilitan la satisfacción de las necesidades y/o deseos de los turistas, a través de su uso.

\section{Servicios Básicos.}

En Catarina existen varios restaurantes y comiderías en los cuales el visitante puede degustar una gran variedad de platillos tanto nacionales como extranjeros.

\section{Restaurantes}

En los diversos establecimientos se ofrece gran variedad de la gastronomía nacional e internacional, donde los turistas pueden degustar todo tipo de platillos ya sea comida tradicional o a la carta, entre los restaurantes más visitados están: Restaurante Libro Café, Restaurante Brisas de Apoyo.

El restaurante "del lago" con capacidad para 350 personas ofrece un variado menú con platos de excelente sabor y banquetes de primera calidad.

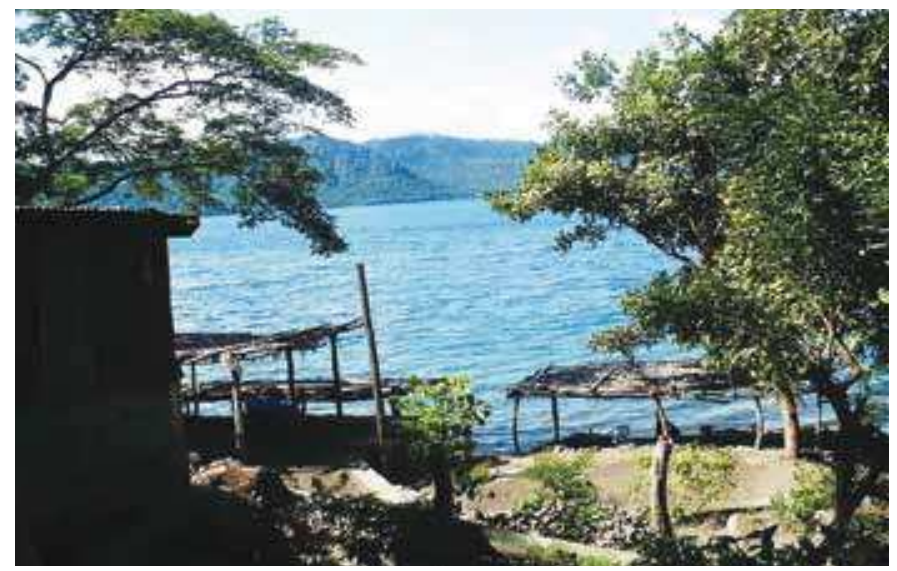

Foto 6. Mirador de la Laguna.

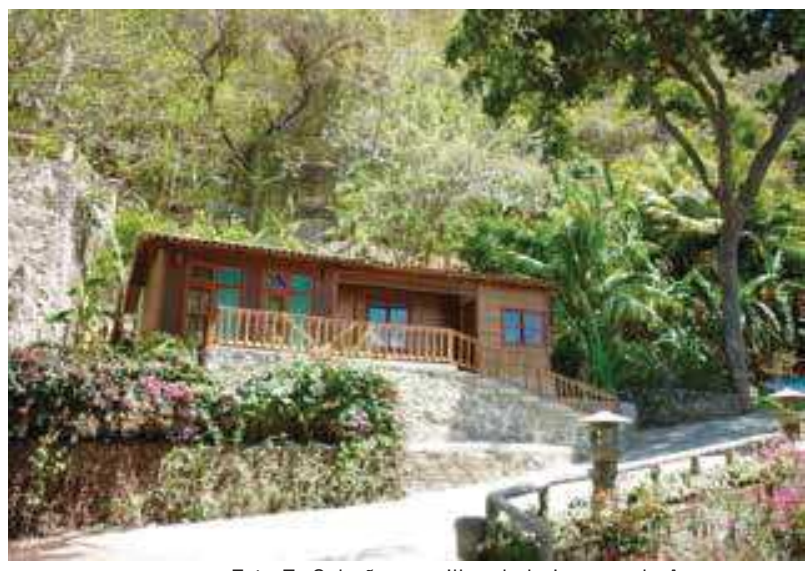

Foto 7. Cabañas a orillas de la Laguna de Apoyo. 
Este gran hotel ofrece actividades variadas como: voleibol acuático, entretenimiento nocturno con baile y karaoke, tours clásicos, masajes relajantes, caminatas ecológicas por el sendero los congos, piscina y área de juegos para niños, dos piscinas de adultos, kayaks, veleros y bicicletas acuáticas y un gran centro de conferencias para 200 personas.

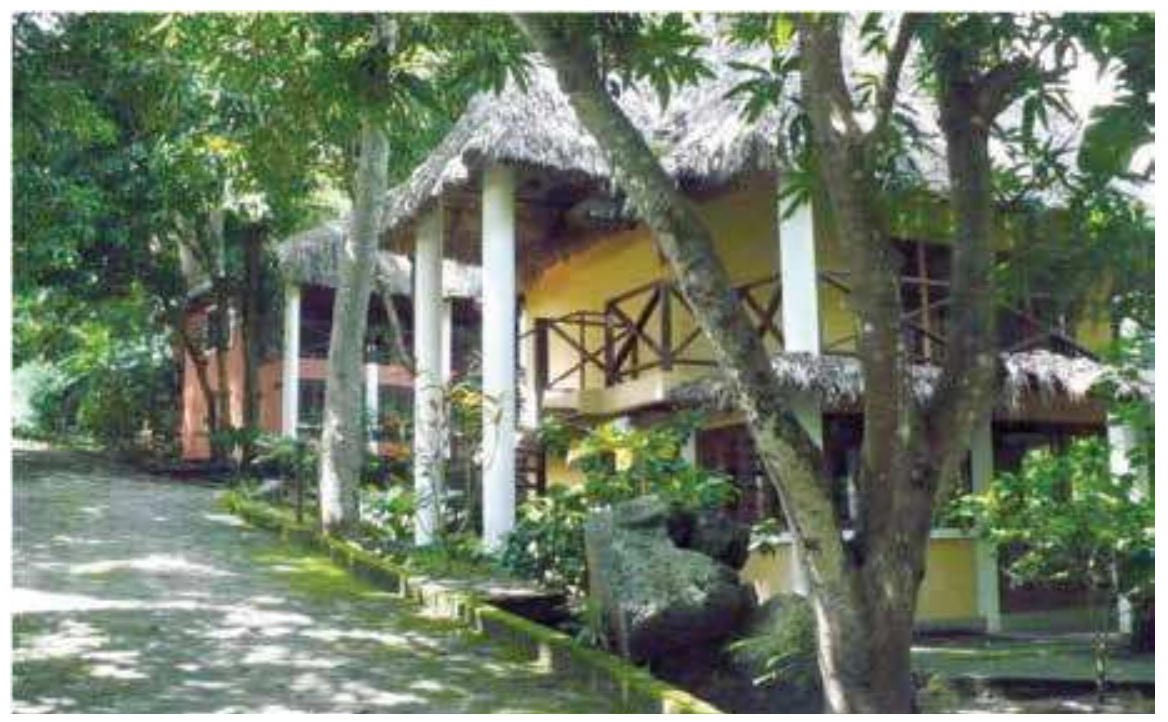

Foto 8. Hotel Norome Resort Villas

\section{Conclusiones}

Catarina es un pueblo pequeño pero muy atractivo debido a los recursos que posee: la laguna de Apoyo con sus miradores y senderos, la variedad de jardines y los talleres artesanales, todos estos componentes constituyen el mayor potencial de atractivos turísticos.

La localidad es muy pequeña pero cuenta con la infraestructura hotelera mínima para garantizar la atención a los visitantes.

Se encuentra ubicada a la orilla del borde de la laguna y a pocos kilómetros de la ciudad de Masaya (6km), Granada y Managua $(45 \mathrm{~km})$.

Recibe un flujo de turistas abundante tanto nacionales como extranjeros principalmente los fines de semana y en la época de verano.
En la localidad hay fuerte demanda de flores y de artesanías, las que son adquiridas por los visitantes y en otros casos también son exportados a los países vecinos.

\section{Referencias Bibliográficas}

Alemán Giselle. TURISMO EN NICARAGUA aportes y desafíos primera parte 2007.

Inventario de la oferta turística del sector urbano del municipio de Catarina el departamento de Masaya 2008.

INTUR, Manual de campo, proceso metodológico de la planificación turística municipal, 2007.

Web grafía

http://es.wikipedia,org/wiki/turista.

http: / / catarinense.wordpress. com/2007/07/17/ reserva-naturallaguna-de-apoyo. 